\title{
Terpolymers for modifying the performance properties of engine oil
}

\author{
Rabab M. Nasser • Nehal S. Ahmed • \\ Amal M. Nassar
}

Received: 26 August 2014/Accepted: 13 October 2014/Published online: 2 December 2014

(C) The Author(s) 2014. This article is published with open access at Springerlink.com

\begin{abstract}
Nine terpolymers consisting of (jojoba: alkylacrylate: $\alpha$-olefins) backbone were synthesized via free radical chain addition polymerization and determined using Fourier transform infrared spectroscopy, proton nuclear magnetic resonance and gel permeation chromatography, for determination of weight average molecular weight. The alkylacrylates used were (dodecylacrylate, tetradecylacrylate and hexadecylacrylate), while $\alpha$-olefins used were (dodecene, tetradecene and hexadecane). The monomers' ratio used for polymerization was $(1: 1: 1)$. It was found that the molecular weight increases with increasing the alkyl chain length of both alkylacrylate and $\alpha$-olefins used. The thermal stability of the synthesized terpolymers was determined using thermal gravimetric analysis and differential scanning calorimetry. The prepared polymers were evaluated as performance modifiers (viscosity index improvers and pour point depressants) for free additives lube oil (SAE-30). It was found that the viscosity index increases with increasing the alkyl chain length, molecular weight and concentration of used terpolymer. The effect of the prepared terpolymers on the pour point of lube oil was studied and it was found that the pour point of lube decreases (more negative) with decreasing the alkyl chain length of both alkylacrylate and $\alpha$-olefin used.
\end{abstract}

Keywords Lube oil additives - Performance modifiers additives · Viscosity index improvers · Pour point depressants Thermal gravimetric analysis

R. M. Nasser ( $₫)$ · N. S. Ahmed · A. M. Nassar

Department of Petroleum Applications, Egyptian Petroleum

Research Institute, Nasr City, Cairo, Egypt

e-mail: rabab_nasser@yahoo.com

\begin{abstract}
Abbreviations
FTIR Fourier transform infrared spectroscopy

${ }^{1} \mathrm{H}-\mathrm{NMR}$ Proton nuclear magnetic resonance

GPC Gel permeation chromatography

TGA Thermal gravimetric analysis

DSC Differential scanning calorimetry
\end{abstract}

\section{Introduction}

It is always implied that the lubrication involves the use of lubricating oil, usually formulated by blending the appropriate quality lubricant base stock and additives. Incidentally, for some applications, such as automotive engine oils and transmission fluids, the additives' manufacturer supplies additives as a package that meets certain industry-specified testing criteria. In other cases, such as metalworking fluids and greases, the additives' supplier furnishes individual additives, which the end user adds to the base stock [1].

Lubricant additives are chemicals, nearly always organic or organometallic, that are added to oils in quantities of a few weight percent to improve the lubricating capacity and durability of the oil. Specific purposes of lubricant additives are improving the wear and friction characteristics by provision for adsorption and extreme pressure (EP) lubrication, improving the oxidation resistance, control of corrosion, control of contamination by reaction products, reducing excessive decrease of lubricant viscosity at high temperatures, and enhancing lubricant characteristics by reducing the pour point and inhibiting the generation of foam [2].

Viscosity index (VI) improvers are long chain, highmolecular weight polymers that function by causing the relative viscosity of an oil to increase more at high temperatures than at low temperatures. Generally, this result is

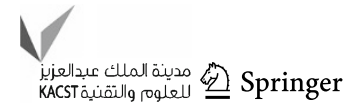


due to a change in the polymer's physical configuration with increasing temperature of the mixture. It is postulated that in cold oil the molecules of the polymer adopt a coiled form so that their effect on viscosity is minimized. In hot oil, the molecules tend to straighten out, and the interaction between these long molecules and the oil produces a proportionally greater thickening effect [3]. Among the principal VI improvers are methacrylate polymers and copolymers, acrylate polymers, olefin polymers and copolymers, and styrene butadiene copolymers. The degree of VI improvement from these materials is a function of the molecular weight distribution of the polymer [3].

Certain high-molecular weight polymers function by inhibiting the formation of a wax crystal structure that would prevent oil flow at low temperatures. Two general types of pour point depressant are used: alkylaromatic polymers adsorb on the wax crystals as they form, preventing them from growing and adhering to each other and polymethacrylates cocrystallize with wax to prevent crystal growth. The additives do not entirely prevent wax crystal growth, but rather lower the temperature at which a rigid structure is formed [3].

Jojoba plant cultivation began in Egypt during the ninth decade of the nineteenth century. The potential importance of jojoba oil for industrial uses has stimulated research on recovery. Jojoba oil has many vital uses such as skincare products, food, high-pressure lubricants; jojoba oil has been much useful application in the field of lubricating oil additives. Sivasankaran et al. [4] showed the suitability of jojoba oil as a component in lubricating oil formulations for two-cycle gasoline engines [5]. Bisht et al. [6] studied the utilization of jojoba oil as an additive for lubricating oil.

In the present work, novel nine terpolymers were prepared via free radical chain addition polymerization, using (1:1:1) molar ratio of (jojoba: alkylacrylate: $\alpha$-olefins) terpolymers. The alkylacrylates used were dodecylacrylate, tetradecylacrylate and hexadecylacrylate while the $\alpha$-olefins used were dodecene, tetradecene and hexadecane. The prepared terpolymers were determined by FTIR, ${ }^{1} \mathrm{H}-\mathrm{NMR}$ and GPC for determination of molecular weight. The thermal analyses of the prepared terpolymers were measured using thermal gravimetric analysis (TGA) and differential scanning calorimetry (DSC) analysis. The prepared additives were evaluated as lube oil viscosity index improvers and pour point depressants.

\section{Materials and methods}

Esterification of acrylic acid with different fatty alcohols

Three esters were prepared via the esterification reaction of 1 mole of acrylic acid (99\%), with dodecanol (98\%), tetradecanol (99\%), and hexadecanol (99\%) separately. The reactions were carried out in a resin kettle in the presence of $0.5 \%$ by wt. $p$-toluene sulphonic acid $(98.5 \%)$, as a catalyst, $0.25 \%$ by wt. hydroquinone (99\%), as inhibitor for the polymerization of acrylic acid, and xylene as a solvent. All reagents were obtained from Sigma-Aldrich. The esterification reactions were carried out under a slow stream of deoxygenated nitrogen. The reactions were agitated using a mechanical stirrer at $500 \mathrm{rpm}$. The reactants, which were mixed with an equal weight of xylene, were heated gradually from room temperature to $130 \pm 5{ }^{\circ} \mathrm{C}$ using a well-controlled thermostat. The extent of reaction was followed by monitoring the amount of liberated water to give products dodecylacrylate, tetradecylacrylate, and hexadecylacrylate [7-9].

Terpolymerization of jojoba with different monomers

Novel nine terpolymers consisting of (jojoba-alkylacrylate- $\alpha$-olefins) were prepared using different alkylacrylates (dodecylacrylate, tetradecylacrylate, and hexadecylacrylate) separately and using different $\alpha$-olefins (99\% 1-dodecene, $97 \%$ 1-tetradecene, and $99 \%$ 1-hexadecene) separately with $(1: 1: 1)$ molar ratio and alternately (Scheme 1). The polymerization was carried out using magnetic stirrer in a 3-neck, round-bottom flask equipped with an efficient condenser, thermometer, and an inlet for the introduction of nitrogen, an efficient weight of benzoyl peroxide was used at the desired temperature $80 \pm 10^{\circ} \mathrm{C}$. When the reaction was completed, the temperature was allowed to reduce to room temperature. Then, the reaction mixture was poured drop by drop in cooled methanol with continuous stirring, filtered off, and dried.

Elucidation of the prepared terpolymers

\section{Spectroscopic analysis}

Infrared spectroscopic analysis Infrared spectra of synthesized esters and jojoba terpolymers were measured using FTIR-Spectrometer Model Type Mattson Infinity Series Bench Top 961 for the purified esters.

${ }^{1} H$-NMR spectroscopic analysis The structure of the synthesized polymeric additives was followed up by proton nuclear magnetic resonance $\left({ }^{1} \mathrm{H}-\mathrm{NMR}\right)$ spectra using a $300 \mathrm{MHz}$ Varion NMR 300 spectrometer using DMSO as a solvent.

Determination of molecular weight of prepared esters and terpolymers

The molecular weights of the prepared esters were determined using a vapor pressure osmometer, while the 
Scheme 1 a "Jojobadodecylacrylate- $\alpha$-olefins" terpolymers. $b$ "Jojoba tetradecylacrylate- $\alpha$-olefins" terpolymers. $c$ "Jojobahexadecylacrylate- $\alpha$-olefins" terpolymers<smiles>C=CCC(C)CCCC(=O)OCC=CCC(C)CC</smiles>

Benzoyl peroxide

$80-90{ }^{\circ} \mathrm{C}, \mathrm{N}_{2}$<smiles>CCCC(CC)C(CC)C(C)CCC(=O)OCC(C)C(C)CC(CC)C(=O)OCC</smiles>

molecular weights of the prepared terpolymers were determined using Agilent GPC, Germany Poly Strogel (Germany), particle size 100, 104, 105 $\mathrm{A}^{\mathrm{o}}$.

Thermal analysis for the prepared terpolymers

TGA and DSC experiments were carried out using Simultaneous Q-600 DSC/TGA (USA). The experiments use $0.01 \mathrm{~g}$ polymer sample, at a heating rate of $10{ }^{\circ} \mathrm{C} \mathrm{min}{ }^{-1}$ from 25 to $600{ }^{\circ} \mathrm{C}$, under a flowing $\left(25 \mathrm{ml} \mathrm{min}^{-1}\right.$ ) nitrogen atmosphere.

Evaluation of the prepared terpolymers as lubricating oil additives

\section{As viscosity index improvers}

The prepared terpolymers were evaluated as viscosity index improvers using free additive base oil (SAE-30) through the viscosity index (VI) test, according to the ASTM D-2,270-93. The Kinematic viscosity of the oil containing the tested compound was determined at 40 and $100{ }^{\circ} \mathrm{C}$. Different concentrations ranging between 0.0 and
3.0 wt.\% were used to study the effect of additive concentration on VI.

As pour point depressants for lube oil

The evaluation of the prepared terpolymers was carried out using the ASTM-D 98-93 for measuring the pour point (PP).

\section{Results and discussion}

Characterization of the prepared esters

\section{Using infrared spectroscopic analysis}

The compositions of the prepared esters were confirmed by IR spectra, From IR spectrum of hexadecylacrylate, which is shown in Fig. 1, one can arrive at the following: no sign for the presence of strong absorption band at $3,200 \mathrm{~cm}^{-1}$ of aliphatic $(-\mathrm{OH})$ group or the characteristic absorption bands of the carboxylic acid. The band for ester group appears at 1,737 and $1,269 \mathrm{~cm}^{-1}$ due to $\mathrm{C}=\mathrm{O}$ of aliphatic 
Fig. 1 IR spectrum of hexadecylacrylate

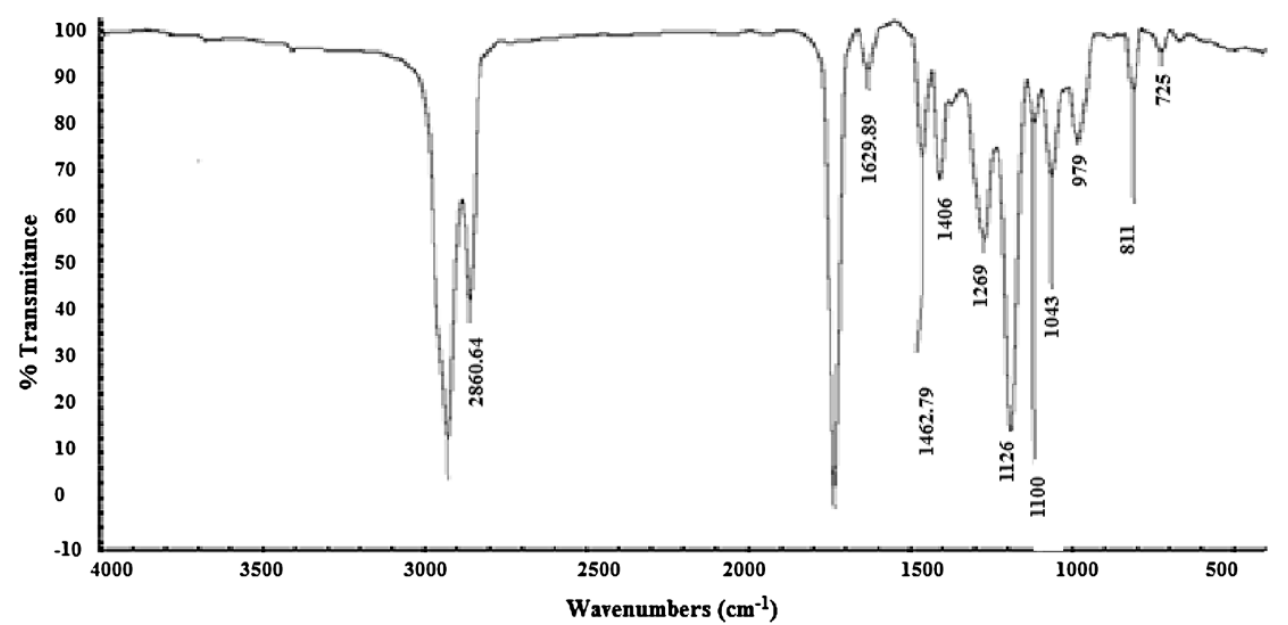

ester and $\mathrm{C}-\mathrm{O}-\mathrm{C}$ stretching, respectively. The band for methylene group $(-\mathrm{CH}=\mathrm{CH}-)$ appears near the band for methyl group, which appears near $1,465 \mathrm{~cm}^{-1}$. The band for methyl group appears near $1,370-1,465 \mathrm{~cm}^{-1}$. The band for $(\mathrm{C}-\mathrm{H})$ aliphatic appears near $2,840 \mathrm{~cm}^{-1}$ and $2,950 \mathrm{~cm}^{-1}$. Disappearance of the strong band at $3,200 \mathrm{~cm}^{-1}$ and the characteristic bands of $(-\mathrm{COOH})$ group indicate that all hydroxyl and carboxyl groups of alcohols and acids, respectively, were consumed in the esterification reaction.

\section{By determination of molecular weight}

It is obvious from the data given in Table 1 that the experimental and theoretical values of the molecular weight are in good accord. This indicates that the reactions were completed successfully.

\section{Characterization of the prepared terpolymers}

The prepared terpolymers were elucidated using different techniques of spectral analysis, FTIR, ${ }^{1} \mathrm{H}-\mathrm{NMR}$, determining molecular weight, and measurement of thermal analysis.

\section{Using infrared spectroscopic analysis}

The spectrum of jojoba oil (Fig. 2) indicates that it contains the following functional groups [11]: ester $(\mathrm{C}=\mathrm{O}$ with

Table 1 Theoretical and experimental molecular weights for the prepared esters

\begin{tabular}{lll}
\hline Ester & Theoretical M.wt. & Experimental M.wt. \\
\hline Dodecylacrylate & 240 & $243 \pm 1$ \\
Tetradecylacrylate & 268.45 & $268 \pm 1$ \\
Hexadecylacrylate & 297.02 & $298 \pm 1$ \\
\hline
\end{tabular}

frequency $1,820-1,660 \mathrm{~cm}^{-1}$ and $\mathrm{C}-\mathrm{O}$ with frequency $\left.1,465-1,375 \mathrm{~cm}^{-1}\right)$.

(Jojoba: alkylacrylate: $\alpha$-olefins) terpolymers were prepared via free radical polymerization using (1:1:1) molar ratios (Scheme 1a-c).

The prepared terpolymers were elucidated using FTIR (Fig. 3) corresponding to (dodecylacrylate-jojoba-dodecene) terpolymer a characteristic peak at $1,737 \mathrm{~cm}^{-1}$ corresponding to $(\mathrm{C}=\mathrm{O})$ group of ester, peak at $1,169 \mathrm{~cm}^{-1}$ corresponding to $(\mathrm{C}-\mathrm{O})$ group of ester and a peak at $2,856 \mathrm{~cm}^{-1}$ corresponding to $(\mathrm{C}-\mathrm{H})$ aliphatic.

\section{Using ${ }^{1} \mathrm{H}-\mathrm{NMR}$}

The structure of the prepared terpolymers was confirmed using ${ }^{1} \mathrm{H}-\mathrm{NMR}$, as in Fig. 4.

${ }^{1} \mathrm{H}-\mathrm{NMR}$ spectrum of (E) terpolymer was found characteristics peak signals corresponding to $\delta-0.865$ for $\left(-\mathrm{CH}_{3}\right)$ of alkylacrylate, $\delta-3.5$ for $\left(-\mathrm{O}-\mathrm{CH}_{2}\right)$ of alkylacrylate, $\delta$-2.254 for $-(\mathrm{CO}-\mathrm{CH})$ of alkyl acrylate, peak signal at $\delta-3.8$ corresponding to $-\left(\mathrm{COO}-\mathrm{CH}_{2}\right)-$ of jojoba oil.

\section{Molecular weight determination}

The weight average molecular weight (Mw), number average molecular weight (Mn), and polydispersity index (PDI) of the prepared terpolymers, their designation and their composition are given in Table 2, which indicates that the molecular weight increases with increasing the alkyl chain length of both alkylacrylate and $\alpha$-olefin used.

\section{Thermal stability of polymers}

The thermal stability of the prepared polymers was investigated (Figs. 5, 6); both integral and differential are shown for all the investigated polymers. It was found that the 
Fig. 2 IR spectrum of jojoba oil

Fig. 3 FTIR spectrum of (dodecylacrylate-jojobadodecene) terpolymer
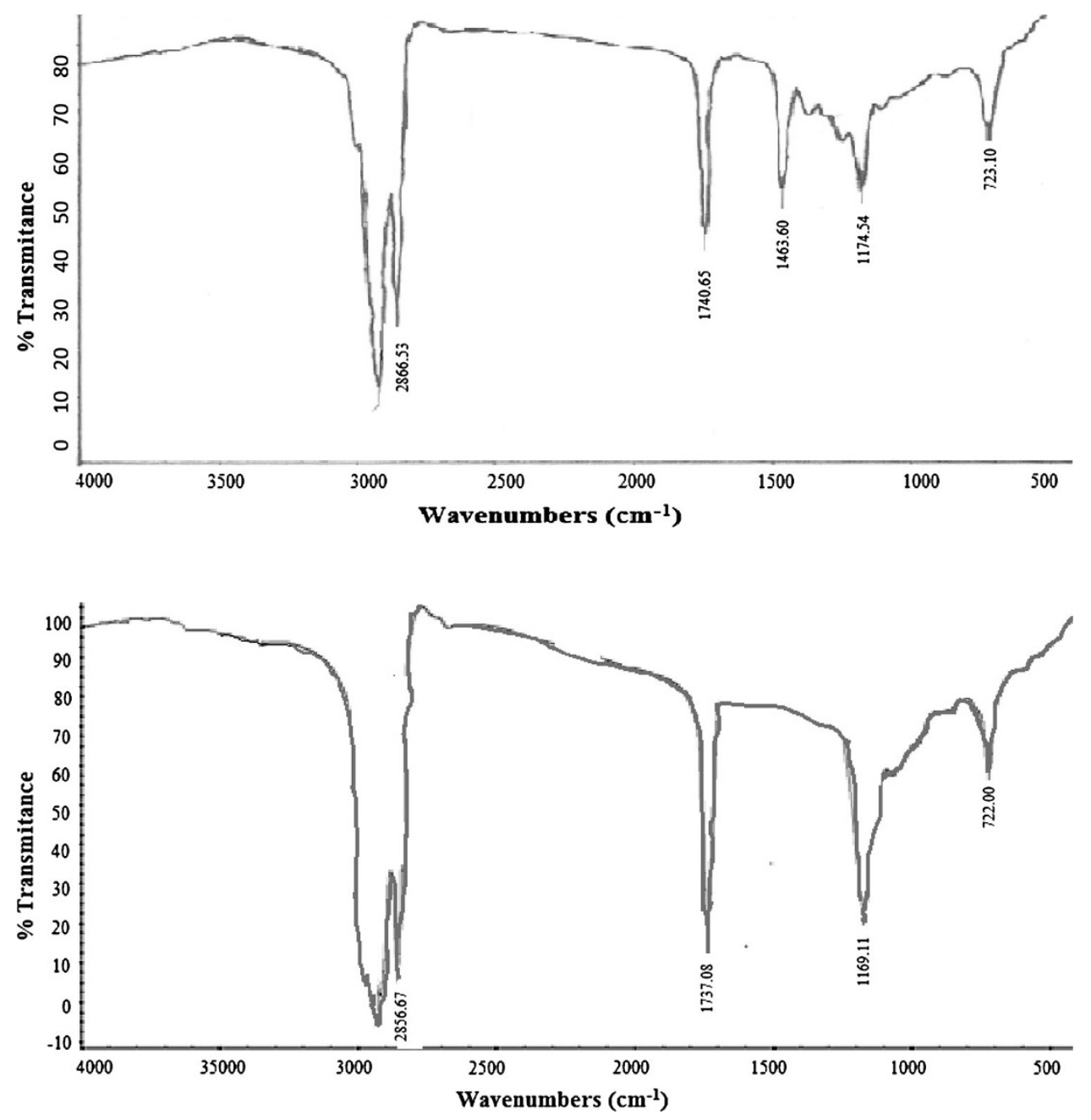

Fig. 4 1H-NMR spectrum of (E) Terpolymer

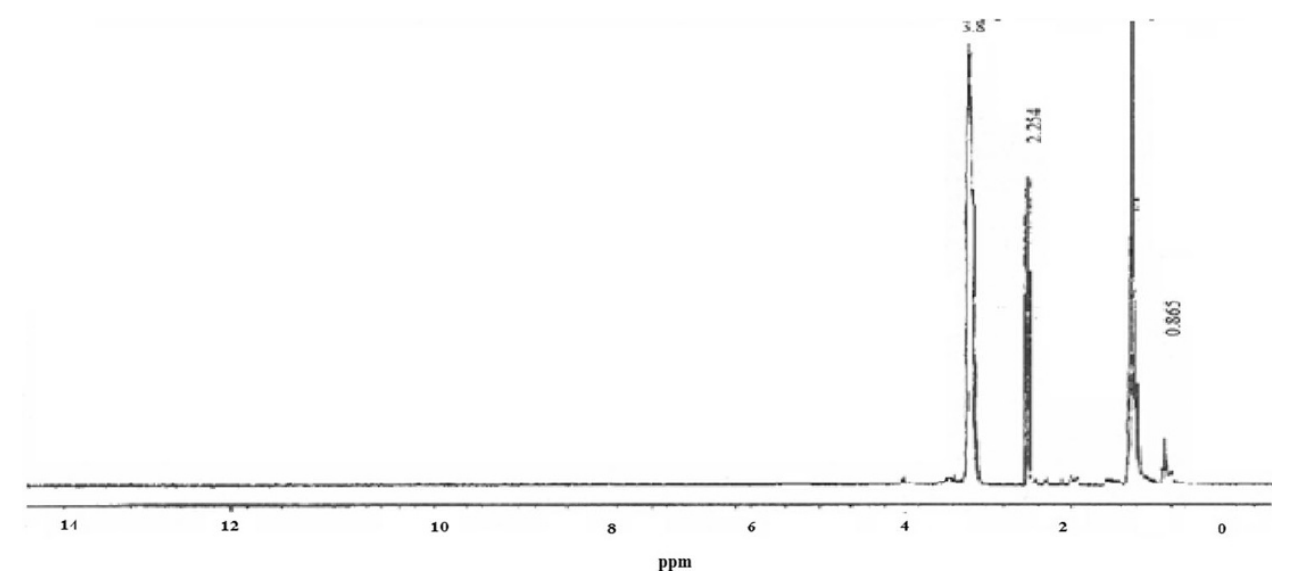

primary degradation for (A-D) terpolymers occurs at $191{ }^{\circ} \mathrm{C}$, with $17.5 \%$ weight loss, while the primary degradation for (E-I) terpolymers occurs at $315^{\circ} \mathrm{C}$, with $9.07 \%$. The complete degradation for all terpolymers occurs at $438{ }^{\circ} \mathrm{C}$ with $95.318 \%$ weight loss. This confirms the suitability of the prepared polymers as lubricating oil additives.
Evaluation of the prepared terpolymers as lube oil additives

As viscosity index improvers for lube oil

The effect of the polymeric compounds as viscosity index improvers depends mainly on the behavior of polymer

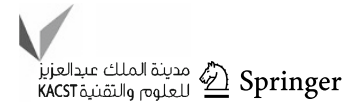


Table 2 Polymers compositions, designations, their number average molecular weight, weight average molecular weight, and polydispersity index

\begin{tabular}{|c|c|c|c|c|}
\hline & Polymer designation & Mn & Mw & PDI \\
\hline A & (Jojoba-dodecylacrylate-1-dodecene) terpolymer & 11,655 & 14,732 & 1.264 \\
\hline B & (Jojoba-odecylacrylate-1-tetradecene) terpolymer & 12,767 & 16,048 & 1.257 \\
\hline $\mathrm{C}$ & (Jojoba-dodecylacrylate-1-hexadecene) terpolymer & 13,564 & 18,270 & 1.347 \\
\hline $\mathrm{D}$ & (Jojoba-tetradecylacrylate-1-dodecene) terpolymer & 15,250 & 22,631 & 1.484 \\
\hline $\mathrm{E}$ & (Jojoba-etradecylacrylate-1-tetradecene) terpolymer & 16,950 & 22,729 & 1.341 \\
\hline $\mathrm{F}$ & (Jojoba-tetradecylacrylate-1-hexadecene) terpolymer & 18,650 & 25,215 & 1.354 \\
\hline G & (Jojoba-hexadecylacrylate-1-dodecene) terpolymer & 20,144 & 27,234 & 1.352 \\
\hline $\mathrm{H}$ & (Jojoba-hexadecylacrylate-1-tetradecene) terpolymer & 22,450 & 30,801 & 1.372 \\
\hline I & (Jojoba-hexadecylacrylate-1-hexadecene) terpolymer & 24,560 & 35,244 & 1.435 \\
\hline
\end{tabular}

molecules in the dispersed phase (base oil). The prepared compounds (A-I) were tested for their effectiveness as viscosity index improvers for the lube oil. In this respect, the kinematic viscosity of the undoped oil, and oil containing different concentrations of the tested additives was determined at 40 and $100{ }^{\circ} \mathrm{C}$ [7-9]. The viscosity index and pour point of the lube oil (SAE-30) and jojoba oil are given in Table 3.

Effect of additive concentration on viscosity index of lube oil

Different concentrations of the prepared additives ranging from $0.25,0.50,1.00,2.00$, and $3.00 \%$ by weight were used to study the effect of the additive concentration on VI; the data are tabulated in Table 3 , which indicates that the VI increases with increasing the concentration of the prepared additives in solution. The viscosity of a particular fluid is not constant, however, but varies with temperature. As oil is heated, its viscosity decreases, and becomes thinner. The polymer-oil interaction at low temperature is minimal but increases as the temperature rises. This interaction of the polymer with the base oil at elevated temperatures increases the effective hydrodynamic volume of the polymer, thereby increasing the effective volume fraction of the viscosity modifier. This, in turn, leads to an increase in lubricant viscosity [10]. The increase in the concentration of the polymer leads to an increase in the total volume of polymer micelles in the oil solution. Consequently, a high concentration of polymer will impart a higher viscosity index than a low concentration of the same polymer [11].

\section{Effect of alkyl chain length of the prepared additives on viscosity index of lube oil}

The effect of alkyl chain length on the efficiency of the prepared additives as viscosity index improvers for lube oil is indicated at Fig. 7, which indicates that the efficiency increases with increasing the alkyl chain length of both alkylacrylate and $\alpha$-olefins. This may be due to the influence of the molecular weight on the effective coil radius and hence the viscosity index [11].

Effect of monomer type on viscosity index of lube oil

The effect of monomer type on viscosity index of free additives lube oil (SAE-30) was studied and it was found that the terpolymer based on jojoba-hexadecylacrylatehexadecene has greater effect as viscosity index improvers than the other terpolymers with lower alkyl chain length (Fig. 8). This may be due to greater molecular weight.

\section{As pour point depressants}

Effect of additive concentration on the pour point of lube oil Different concentrations of prepared compounds (A-I) ranging from $0.25,0.5,1.00,2.00$ and $3.00 \%$ by weight were tested as pour point depressants and experimental data are tabulated in Table 3, which indicates that the prepared compounds are efficient as pour point depressants and the efficiency increases by decreasing the concentration of the prepared additives. This is explained by that solvation power of any solvent decreases with decreasing temperature and vice versa. This reduction in solvation power becomes more obvious when the molecular weight of the solute and its concentration increases [11].

\section{Effect of alkyl chain length on pour point of lube oil}

The effect of alkyl chain length on the efficiency of the prepared additives as pour point depressants was studied, as in Fig. 9; it was found that terpolymer which prepared from jojoba-dodecylacrylate-dodecene is the most efficient terpolymer as pour point depressants. This may be due to lower molecular weight. 
Fig. 5 TGA-analysis of (A-I) terpolymers
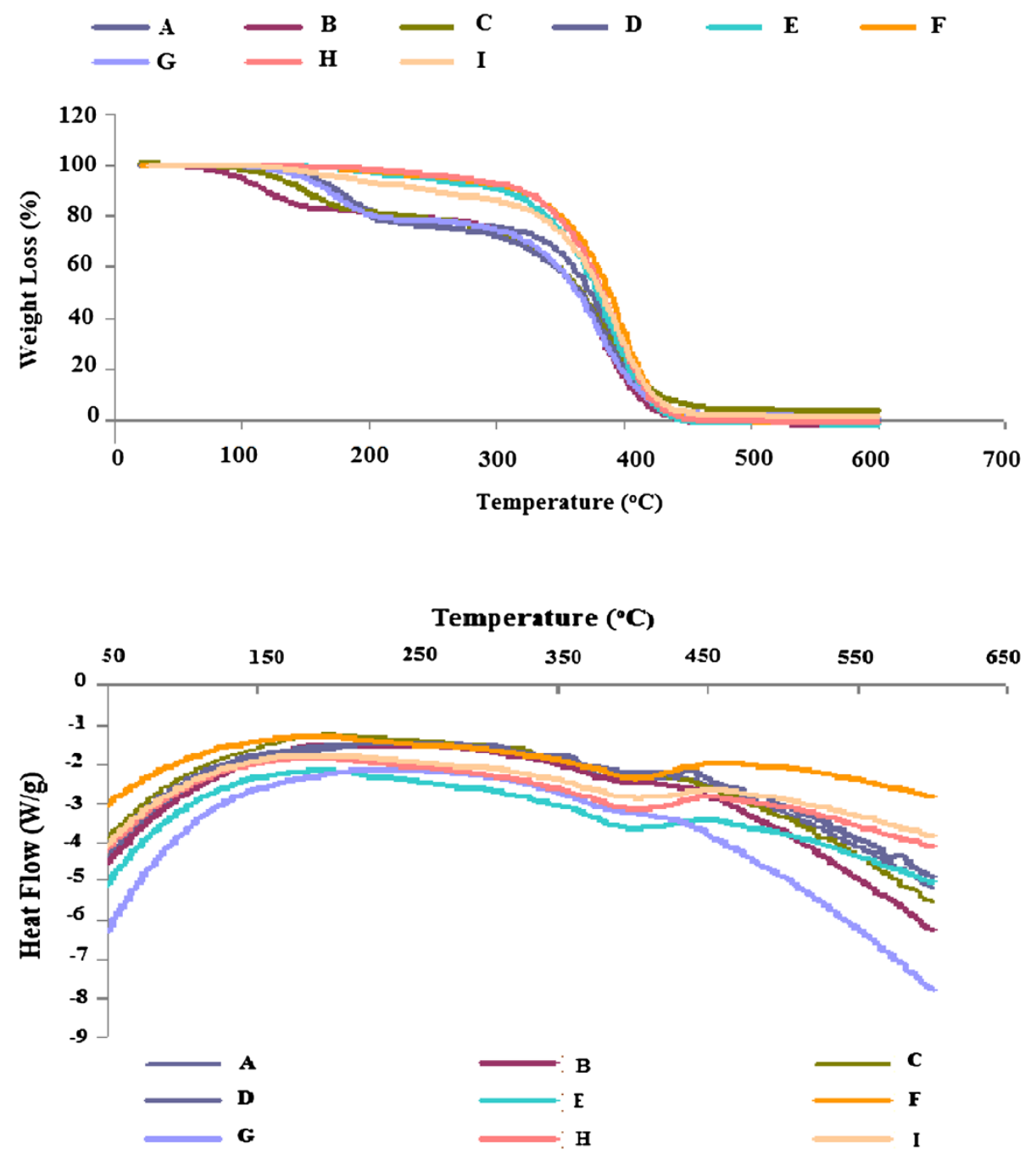

\begin{tabular}{|c|c|c|c|c|c|c|c|c|c|c|c|}
\hline \multirow{2}{*}{\multicolumn{2}{|c|}{ Physical Property }} & \multicolumn{5}{|c|}{ Viscosity Index (VI) } & \multicolumn{5}{|c|}{ Pour Point $\left({ }^{\circ} \mathrm{C}\right)$} \\
\hline & & $0.25 \%$ & $0.50 \%$ & $1.00 \%$ & $2.00 \%$ & $3.00 \%$ & $0.25 \%$ & $0.50 \%$ & $1.00 \%$ & $2.00 \%$ & $3.00 \%$ \\
\hline \multirow{2}{*}{\multicolumn{2}{|c|}{$\begin{array}{c}\text { Lube ollSAE-30 } \\
\text { Jojoba oll }\end{array}$}} & \multicolumn{5}{|c|}{98} & \multicolumn{5}{|c|}{0} \\
\hline & & & & 140 & & & & & 9 & & \\
\hline \multirow{9}{*}{ 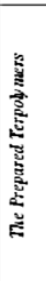 } & $A$ & 104 & 106 & 110 & 114 & 116 & -12 & -12 & -9 & -9 & -6 \\
\hline & $B$ & 106 & 108 & 114 & 116 & 118 & -12 & -12 & -9 & .9 & -6 \\
\hline & $C$ & 106 & 110 & 116 & 118 & 122 & .9 & .9 & -9 & -6 & -6 \\
\hline & $D$ & 106 & 112 & 118 & 120 & 125 & -9 & .9 & -6 & -6 & -3 \\
\hline & $E$ & 108 & 116 & 120 & 122 & 126 & .9 & .9 & -6 & -6 & -3 \\
\hline & $F$ & 114 & 120 & 122 & 124 & 127 & .9 & .9 & -6 & .6 & -3 \\
\hline & $G$ & 116 & 122 & 124 & 126 & 129 & -9 & .9 & -6 & .6 & -3 \\
\hline & $H$ & 116 & 122 & 124 & 126 & 130 & .6 & .6 & .6 & -3 & -3 \\
\hline & $I$ & 118 & 122 & 124 & $12 \mathrm{~s}$ & 133 & -6 & .6 & -3 & -3 & -3 \\
\hline
\end{tabular}

Table 3 Viscosity index and pour point of lube oil (SAE-30), jojoba oil and lube oil treated with the prepared terpolymers
Fig. 6 DSC-analysis of (A-I) terpolymers
Effect of monomer type The effect of monomer type on the efficiency of the prepared additives as pour point depressants for lube oil (SAE-30) was studied, as in Fig. 10; it was found that terpolymer which was prepared from jojoba-dodecylacrylate-dodecene is the most efficient terpolymer as pour point depressants. This may be due to lower molecular weight.

\section{Conclusion}

In this article, with the aim of modifying the performance properties of engine oil, nine terpolymers consisting of (jojoba: alkylacrylate: $\alpha$-olefins) backbone were synthesized via free radical chain addition polymerization. The alkylacrylates used were (dodecylacrylate, tetradecylacrylate and 
Fig. 7 Effect of alkyl chain length of (A-I) terpolymers on VI of lube oil (SAE-30)

Fig. 8 Effect of monomer type of (A-I) terpolymers on VI of lube oil (SAE-30)

Fig. 9 Effect of alkyl chain length of (A-I) terpolymers on PP of lube oil (SAE-30)
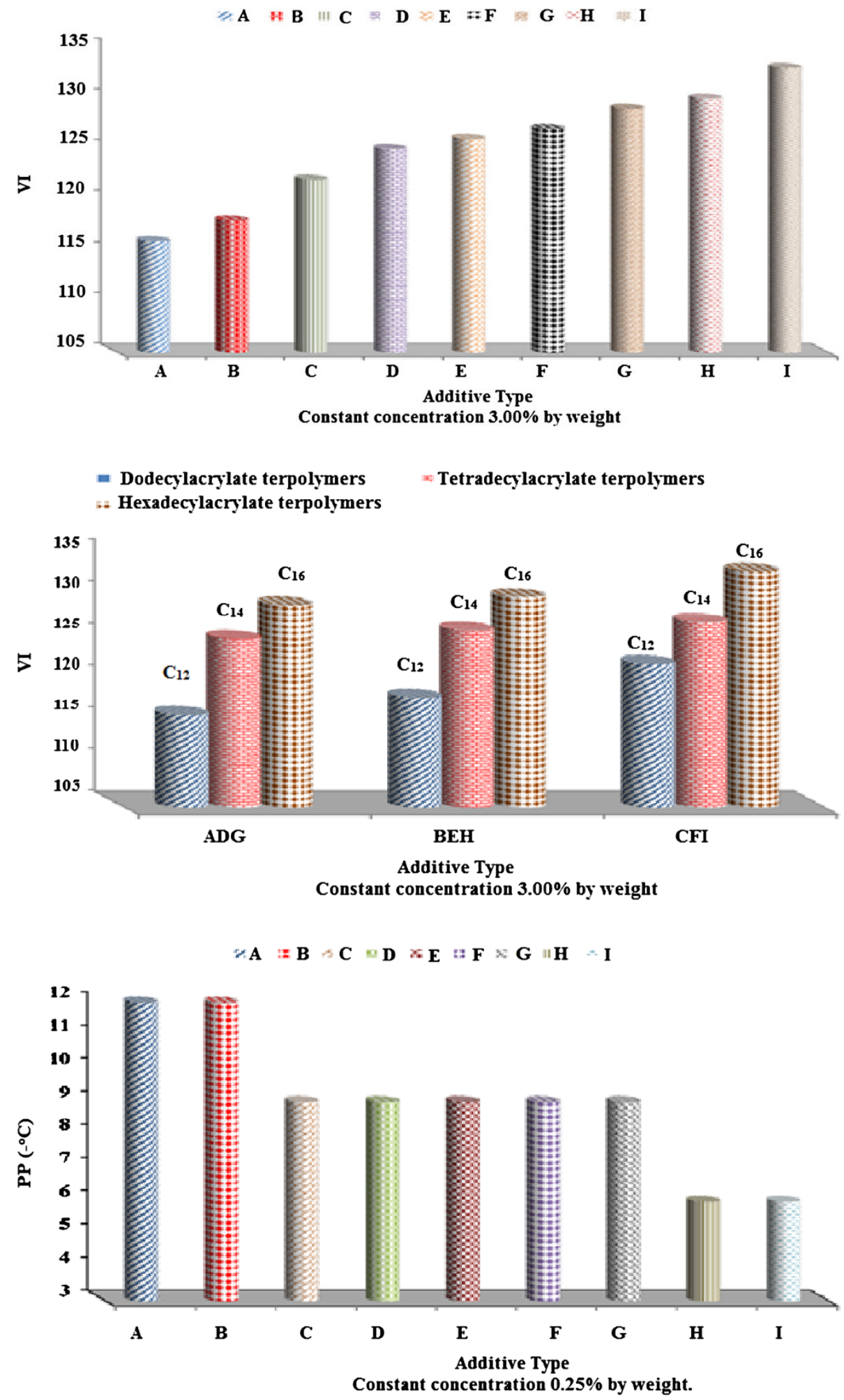

hexadecylacrylate), while $\alpha$-olefins used were (dodecene, tetradecene and hexadecane. The monomers' ratio used for polymerization was $(1: 1: 1)$ and they were characterized using FTIR, ${ }^{1} \mathrm{H}-\mathrm{NMR}$ and GPC, for determination of weight average molecular weight. It was found that the viscosity index increases with increasing the alkyl chain length, molecular weight and concentration of used terpolymer. At the same time, the pour point of lube oil decreases (more negative) with decreasing the alkyl chain length of both alkylacrylate and $\alpha$-olefin used. The thermal stability of the 
Fig. 10 Effect of monomer type of (A-I) terpolymers on VI of lube oil (SAE-30)

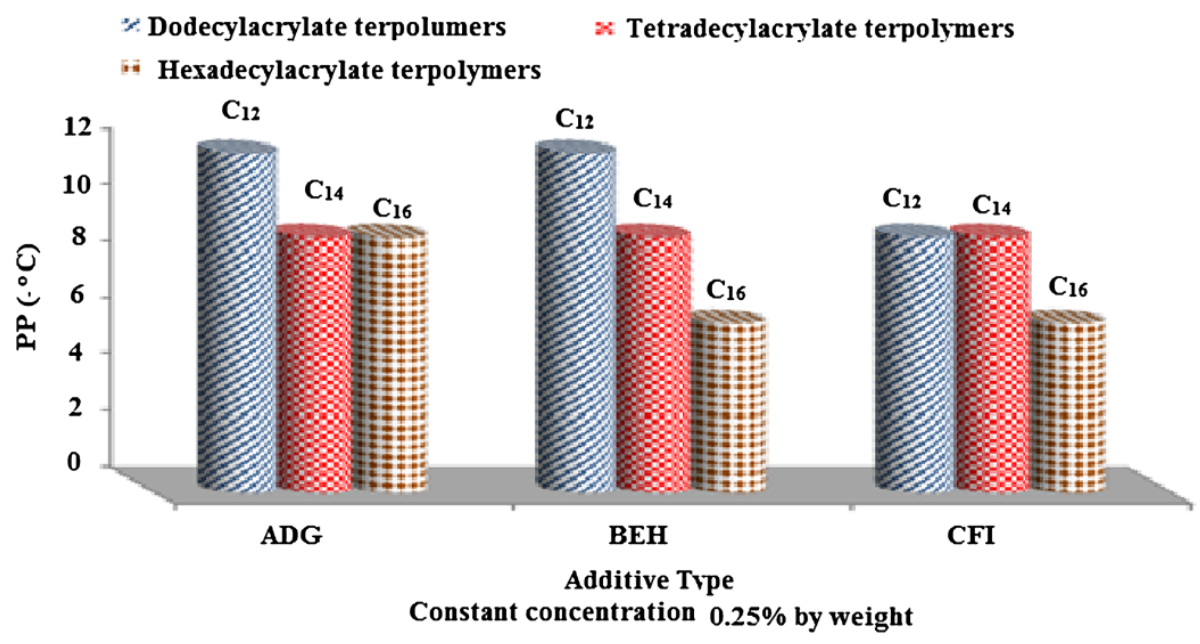

synthesized terpolymers was determined using TGA and DSC. The prepared terpolymers proved its efficiency as lubricating oil additives under severe conditions.

Acknowledgments The authors acknowledge the Egyptian Petroleum Research Institute for its financial and technical support.

Open Access This article is distributed under the terms of the Creative Commons Attribution License which permits any use, distribution, and reproduction in any medium, provided the original author(s) and the source are credited.

\section{References}

1. Syed QR (2009) A comprehensive review of lubricant chemistry, technology, selection, and design. ASTM International, West Conshohocken, pp 100-211

2. Gwidon WS, Andrew WB (2006) Engineering tribology, 3rd edn. Elsevier, USA, p 82

3. Pirro DM (2001) Lubrication fundamentals, 2nd edn. Marcel Dekker, New York, p 31
4. Sivasankaran GA, Bisht RPS, Jain VK, Gupta M, Sethuramiah A, Bhatia VK (1988) ojoba-oil-based two-storke gasoline engine lubricant. Tribol Int 21:327-333

5. Allawzi M, Abu-Arabi MK, Al-zoubi HS, Tamimi A (1998) Physicochemical characteristics and thermal stability of Jordanian jojoba oil. JAOCS 75(1):57-62

6. Bisht RPS, Sivasan Karan GA, Bhatia VK (1993) Additive properties of jojoba oil for lubricating formulations. Wear 161:193-197

7. Ahmed NS, Nassar AM, Nasser RM, Khattab AF, Abdel-Azim AAA (2008) Synthesis and evaluation of some polymeric compounds as pour point depressants and viscosity index improvers for lube oil. Pet Sci Technol 26:1390-1402

8. Ahmed NS, Amal MN, Rabab MN, Khattab AF, Abdel-Azim AAA (2012) J Dispers Sci Technol 33:668-675

9. Ahmed NS, Nassar AM, Nasser RM, Abdel Raouf ME, El-Kafrawy AF (2014) Novel terpolymers as pour point depressants and viscosity modifiers for lube oil. Pet Sci Technol 33:668-675

10. Rizvi SQA (1992) Lubricant additives and their functions. In: Friction, lubrication, and wear technology. ASM handbook, vol 18, 10th edn. ASM International, Material Park, pp 98-112

11. Rabab MN (2012) Ph.D. thesis, The behavior of some acrylate copolymers as lubricating oil additives; Ain Shams University, Cairo, Egypt 